\title{
Knockdown of Long Non-coding RNA TUG1 Suppresses Osteoblast Apoptosis in Particle-induced Osteolysis by Up-regulating BMP-7
}

\author{
Bin $\mathrm{Wu}^{\mathrm{a}, \# \text {, Yang Liu }} \mathrm{L}^{\mathrm{b}, * \#}$ \\ ${ }^{a}$ Department of Orthopaedics, TongLiao City Hospital, Tongliao, Neimenggu 028050, China. \\ ${ }^{b}$ Department of Orthopedics, Affiliated Hospital of inner Mongolia University for the nationalities, No 1742, HuoLinHe Street, Tongliao, \\ Neimenggu 028050, China.
}

\begin{abstract}
Background: Periprosthetic osteolysis may lead to the failure of hip arthroplasty. The role of long non-coding RNA TUG1 in inducing periprosthetic osteolysis after hip arthroplasty remains unknown.

Methods: Clinical tissues were obtained from the patients undergoing hip arthroplasty. Mouse osteoblast cell line MC3T3-E1 was simulated by CoCrMo metal particles (CoPs). Real-time PCR was performed to determine the expression of TUG1 and BMP-7. Western blot was performed to determine the BMP-7 protein expression. Cell apoptosis was determined using flow cytometry. RNA pull-down was performed to determine the interaction between TUG1 and BMP-7. In vivo experiments were performed to verify the role of TUG1.

Results: Overexpressed TUG1 and down-regulated BMP-7 was observed in both clinical periprosthetic tissues and CoPs-stimulated osteoblasts. Knockdown of TUG1 significantly inhibited the apoptosis of CoPs-stimulated osteoblasts by targeting BMP-7. In vivo experiments verified that knockdown of TUG1 increased bone mineral density of the mouse osteolysis model.

Conclusion: Knockdown of TUG1 inhibited the apoptosis of CoPs-stimulated osteoblasts by negatively regulating BMP-7, which might provide a new insight in treating periprosthetic osteolysis after hip arthroplasty.

Keywords: periprosthetic osteolysis; TUG1; BMP-7; osteoblast apoptosis
\end{abstract}

\section{INTRODUCTION}

Artificial joint replacement (AJR) is widely used in the treatment of end-stage arthritis, rheumatoid arthritis, and femoral neck fracture, and significantly decreased joint pain and restore joint function ${ }^{[1]}$. It has been reported that aseptic loosening and periprosthetic osteolysis is the major complication in hip arthroplasty, and could cause long-term failure of joint replacement ${ }^{[2]}$. Previous studies have reported that the inflammatory mediators and cell apoptosis are involved in the failure of hip arthroplasty ${ }^{[3,4]}$.

Long non-coding RNA (lncRNA) is a set of non-coding RNA with the length of more than $200 \mathrm{nt}$, and plays an important role in various physiology processes, such as cell apoptosis, epigenetic modification and inflam-

\footnotetext{
"These authors contributed equally.

*Corresponding author: Yang Liu

Mailing address: Department of Orthopedics, Affiliated Hospital of Inner Mongolia University for the nationalities, No 1742, HuoLinHe Street, TongLiao, Neimenggu 028000,China.

E-mail: Liuyang0919@126.com

TEL: +86-0475-8267850

Received: 2 October 2018 Accepted: 13 December 2018
}

mation. Mounting studies have reported that lncRNAs mediate the pathophysiological mechanism of hip arthroplasty. For example, lncRNA DANCR mediated osteoblast differentiation in regulating FOX01 in total hip arthroplasty ${ }^{[5]}$. LncRNA PRNCR1 regulated osteogenic differentiation and contributed to osteolysis after hip replacement in regulating CXCR4 ${ }^{[6]}$. Taurine-upregulated gene 1 (TUG1), located on the chromosome 22q12, is a 7.1-kb lncRNA ${ }^{[7]}$. TUG1 was first identified as a transcript factor, and it was recently shown as a negative prognostic factor in various diseases, such as osteosarcoma ${ }^{[8]}$ and urothelial carcinoma of the bladder ${ }^{[9]}$. However, the role of TUG1 in inducing periprosthetic osteolysis after hip arthroplasty remains unknown.

Bone morphogenetic proteins (BMPs), belongs to the transforming growth factor (TGF) superfamily, and served as the regulators in osteoblast differentiation of multipotent stem cells ${ }^{[10]}$. BMP-7 is the member of BMPs, and was primarily recognized as the osteogenetic factor, and plays an important role in cell growth, proliferation, and apoptosis, as well as the physiological process of tumor formation ${ }^{[11]}$. It has been reported that miR-542-3p could significantly suppress the proliferation and differentiation of osteoblast via inhibiting 
A

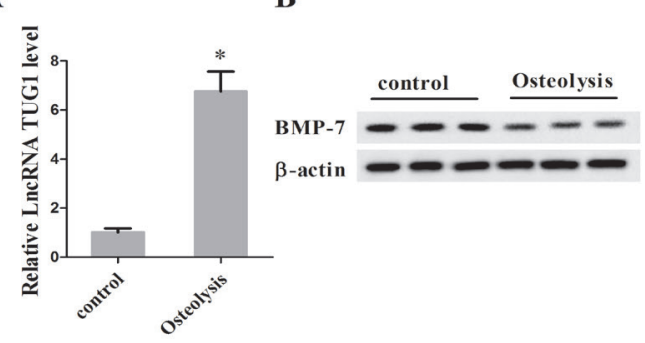

Figure 1. The expression of TUG 1 and BMP-7 in periprosthetic and normal tissues. (A) Real-time PCR was performed to determine the expression of TUG1; (B) Western blot was used to determine the protein level of BMP-7. ${ }^{*} \mathrm{p}<0.05$ vs control.

BMP-7 ${ }^{[12]}$

In the present study, we are attempting to explore the potential role of TUG1 and BMP-7 in periprosthetic osteolysis after hip arthroplasty. We found that TUG1 was increased, while BMP-7 was decreased in clinical periprosthetic tissues. In vitro and in vivo experiments were performed to explore the potential mechanism of TUG1 and BMP-7 in osteolysis. The present study suggested that knockdown of TUG1 suppressed osteoblast apoptosis in particle-induced osteolysis by up-regulating BMP-7.

\section{MATERIALS AND METHODS}

\section{Patients and materials collection}

Total 20 patients who received reoperation of hip arthroplasty in TongLiao City Hospital from January of 2015 to December of 2017 were enrolled in this study. The periprosthetic tissues and the surrounding normal tissues were obtained. Briefly, a thin surface layer of the tissue covering the inner side of the joint capsule was collected after the incision of the hip joint capsule. This layer was designated as the periprosthetic tissue, because it was in a direct physical contact with the implant and prosthetic by-products (wear particles, metal ions, and so forth) contained in the joint fluid. The tissue lying above the periprosthetic tissue was designated as the surrounding normal tissue. The tissue samples were placed into sterile containers separately and deeply frozen at $-80^{\circ} \mathrm{C}$ immediately after sampling. This study was approved by TongLiao City Hospital, and all participants signed the informed consent before the participation.

\section{Cell culture}

CoCrMo metal particles (CoPs), with a diameter of 1.67 $\pm 1.18 \mu \mathrm{m}$, were purchased from Sandvik (Stockholm, Sweden). CoPs were washed in $75 \%$ ethanol solution at room temperature for $36 \mathrm{~h}$ and suspended in phosphate buffered saline (PBS) for the following study. The mouse osteoblast cell line MC3T3-E1 was purchased from American Type Culture Collection (ATCC). The osteoblasts were cultured in Alpha Minimum Essential Medium with ribonucleosides, deoxyribonucleosides, $2 \mathrm{mM}$ L-glutamine and $1 \mathrm{mM}$ sodium pyruvate as well as $10 \%$ fetal bovine serum without ascorbic acid at 37 ${ }^{\circ} \mathrm{C}$ for $24 \mathrm{~h}$. The CoPs $(200 \mu \mathrm{g} / \mathrm{ml})$ were pretreated with ultrasound for $20 \mathrm{~min}$ and then co-cultured with the MC3T3-E1 cells for $20 \mathrm{~h}$.

\section{Real-time PCR}

Total RNAs were isolated from cells or tissues using TRIZOL reagent (Invitrogen). The quantified RNA was reverse-transcripted into cDNA using the BeyoRT ${ }^{\mathrm{TM}}$ II cDNA Kit (Beyotime, China) according to the manufacturer's instruction. cDNA samples were used as template for real-time PCR. The reactions were carried out using the StepOnePlus Real-Time PCR system (Applied Biosystems) in an ABI illumina instrument. The relative expression level of mRNA was calculated using the $2^{-\Delta \Delta C t}$ method.

\section{Western blot}

Cells or tissues were lysed using lysis buffer, and the proteins were isolated after centrifuged at 13,000 rpm for 20 min at $4{ }^{\circ} \mathrm{C}$. The BCA method was used to quantify the protein. After the SDS-PAGE, the separated proteins were transferred to the PVDF membrane and incubated using the primary antibodies at $4{ }^{\circ} \mathrm{C}$ for 24 $h$. The membrane was then incubated using the second antibody at room temperature for $1 \mathrm{~h}$. The protein
A

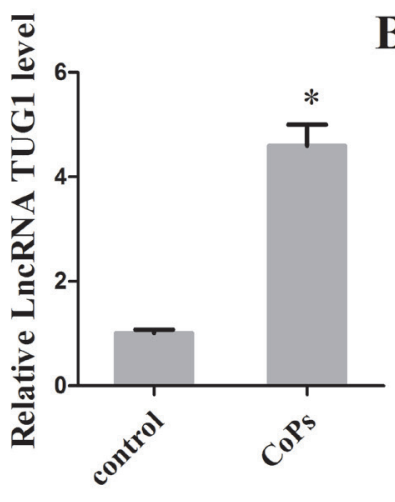
B

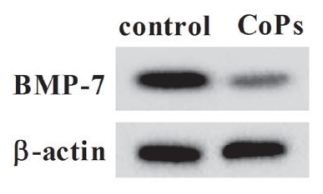
Figure 2. The expression pattern of TUG1 and BMP- 7 in osteoblasts. The MC3T3-E1 cells were stimulated using CoPs to mimic osteolysis; cells were divided into two groups: control and CoPs. (A) Real-time PCR was performed to determine the expression of TUG1; (B) Western blot was used to determine the protein level of BMP-7. ${ }^{*} \mathrm{p}<0.05$ vs control. 
A B

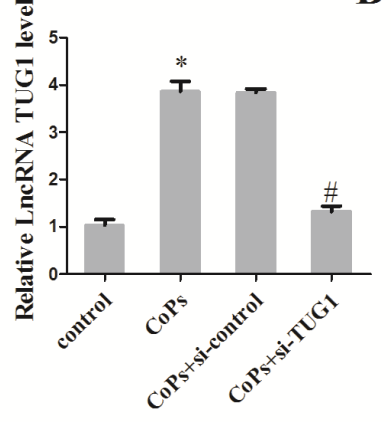

B

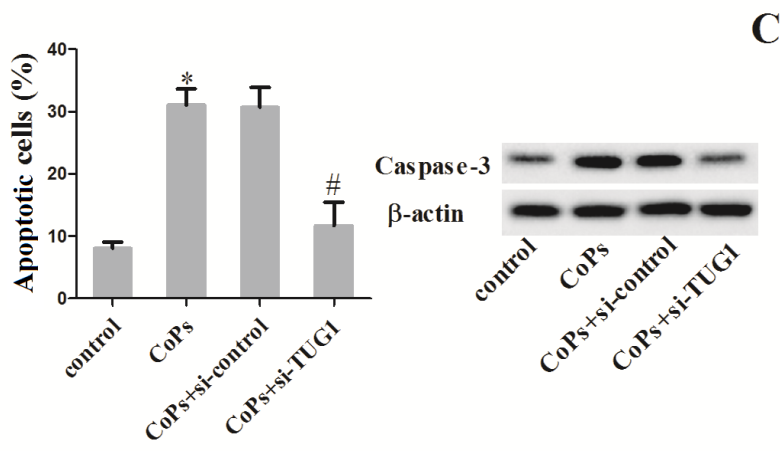

C

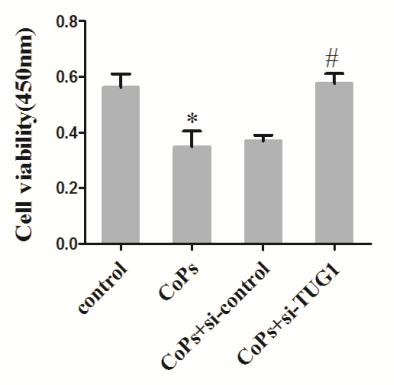

Figure 3. Role of TUG1 on particle-stimulated osteoblast apoptosis. The CoPs-stimulated MC3T3-E1 cells were transfected with siTUG1. (A) The expression of TUG1 was determined by real-time PCR; (B) The expression of TUG1 was determined by real-time PCR; (C) Cell viability was detected by MTT assay. ${ }^{*} \mathrm{p}<0.05$ vs control; $\# \mathrm{p}<0.05$ vs CoPs+si-control.

bands were visualized using ECL chemiluminescence.

\section{Cell transfection}

Cells were seeded in six-well plates and transfected with si-TUG1 or the negative control by Lipofectamine 2000 (Invitrogen) when grew to about 70\% confluence. The lentiviral vectors were purchased from GenePharma Co., Ltd. The transfection efficiencies were examined by qRT-PCR.

\section{RNA pull-down}

For testing the interaction between TUG1 and BMP-7, RNA pull-down was performed using Pierce Magnetic RNA-Protein Pull-Down Kit (Termofsher, CA) in accordance with the manufacturer's instructions. Briefly, the total RNA was extracted from cells, and the magnetic beads were incubated with probes for biotin-labeled TUG1. The relative expression levels were analyzed by real-time PCR.

\section{Cell apoptosis}

Cell apoptosis was determined using flow cytometry. Briefly, cells were stained with annexin $\mathrm{V}$ and propidium iodide (PI) using annexin V-FITC/PI apoptosis detection kits (Beyotime, Shanghai, China). After incubation for $15 \mathrm{~min}$, the cells were examined by flow cytometry (FACScan; BD Biosciences).

\section{Cell proliferation assay}

Cells were plated in 96-well plates $\left(2 \times 10^{5}\right.$ cells $/ \mathrm{mL}$, $100 \mu \mathrm{L} /$ well). After $48 \mathrm{~h}$, cell proliferation and viability were examined using the MTT assay. All experiments were performed in triplicate.

\section{Mouse model establishment}

A total of 16 C57BL/J6 mice was purchased from the Experimental Animal Center of Chinese academy of sciences (Shanghai, China). The mice were anesthetized with Ketamine (70 mg/kg) and Xylazine (5 mg/ $\mathrm{kg}$ ) by intraperitoneal injection, and were fixed in the prone position. The skin was disinfected by scrubbing three times, topically with $0.5 \%$ iodophors. Then a 1.0

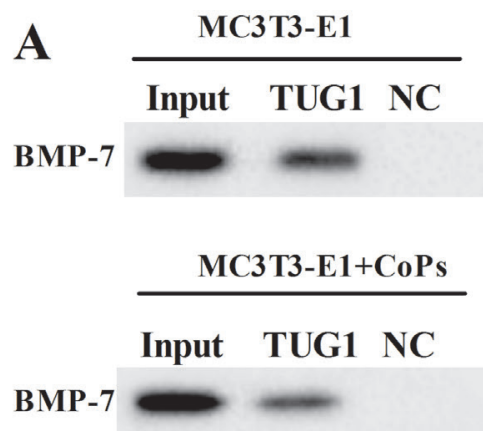

Figure 4. The relationship between TUG1 and BMP-7. (A) RNA pull-down was performed to determine the interaction between TUG1 and BMP-7. (B) The protein level of BMP-7 in CoPs-stimulated MC3T3-E1 cells were determined by western blot. 

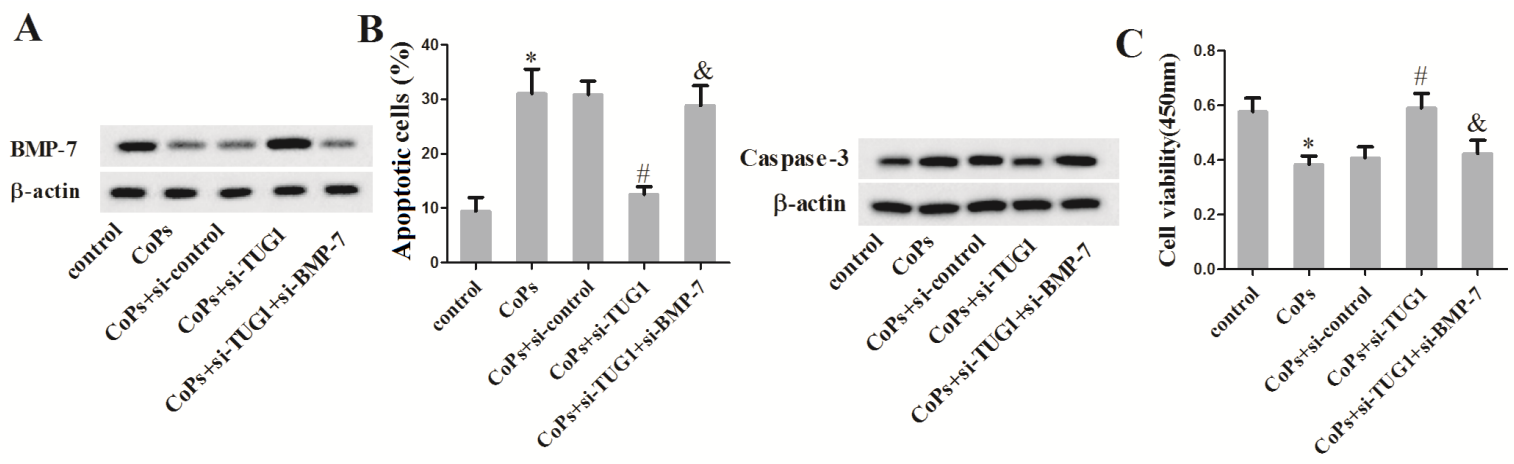

Figure 5. Role of TUG1 and BMP-7 on particle-stimulated osteoblast apoptosis. The CoPs-stimulated MC3T3-E1cells were transfected with si-TUG1 or co-transfected with si-TUG1 and si-BMP-7. (A) Western blot was used to determine the expression of BMP7; (B) Cell apoptosis was detected by flow cytometry and western blot; (C) MTT assay was used to detect cell viability. *p<0.05 vs control; \#p<0.05 vs CoPs+si-control; \&p<0.05 vs CoPs+si-TUG1.

$\mathrm{cm} \times 1.0 \mathrm{~cm}$ area of periosteum was exposed by making a $10 \mathrm{~mm}$ midline sagittal incision over the calvarium that was anterior to the line connecting both external ears. The experimental animals received $30 \mathrm{ml}$ of the CoPs powder suspension, and the control animals received no particles (sham group). The incision was closed using 4-0 nylon thread. The lentivirus carrying si-TUG1 or si-control was injected as a single subcutaneous injection at the surgery site with the indicated dose.

\section{Bone mineral density determination}

Bone mineral density was determined after 14 days post operation. The bone tissues isolated from the osteolysis mice were washed using PBS. Bone mineral density (BMD) was measured using a micro-CT scanner (u80, Scanco Medical AG, Switzerland) according to the manufacturer's instructors ${ }^{[3]}$. Data were analyzed by Advance Bone Analysis Software (GE Health Care Co).

\section{Statistical analysis}

All data were presented as mean \pm SD. Statistical analysis was performed using Student's t-test or variance (ANOVA) analysis. The p values $<0.05$ were considered statistically significant. All experiments were repeated for three independent times.

\section{RESULTS}

Up-regulated TUG1 and down-regulated BMP-7 in periprosthetic tissues and particle-stimulated osteoblasts

To determine the expression of TUG1 and BMP-7 in periprosthetic tissues, the real-time PCR and western blot were performed. As is presented in Figure 1, the RNA level of TUG1 was significantly increased, but the protein level of BMP-7 was notably decreased in peri- prosthetic wear tissues in comparison with the normal tissues. We also stimulated the MC3T3-E1 cells with CoPs, and the results indicated that the expression of TUG1 was dramatically increased, although the expression of BMP-7 was decreased in CoPs-stimulated MC3T3-E1 cells (Figure 2).

\section{Knockdown of TUG1 suppressed cell apop- tosis of particle-stimulated osteoblasts}

To explore whether the expression of TUG1 affects cell apoptosis of particle-induced osteoblasts, we transfected the particle-induced MC3T3-T1 cells with si-TUG1, thus reducing the TUG1 level (Figure 3A). Then we detected the rate of apoptotic cells and found that the CoPs-stimulated osteoblast apoptosis and the expression of apoptosis-related protein caspase- 3 were both increased, but were reversed by knockdown of TUG1 (Figure 3B). In addition, si-TUG1 restored the defect of cell viability of CoPs-stimulated osteoblast using MTT assay (Figure 3C).

\section{The target relationship between TUG1 and BMP-7}

The interaction between TUG1 and BMP-7 was confirmed by RNA pull-down assay. The results revealed that BMP-7 was an RNA pull-down compound of TUG1 in the MC3T3-E1 cells with or without CoPs stimulation (Figure 4A). Then the CoPs-stimulated MC3T3-T1 cells were transfected with si-TUG1, and the protein level of BMP-7 was significantly enhanced (Figure 4B). These data demonstrated the target relationship between TUG1 and BMP-7.

\section{Knockdown of TUG1 suppressed parti- cle-induced osteoblast apoptosis by pro- moting BMP-7 expression}

To explore the potential mechanism of TUG1 and BMP- 
7 in cell apoptosis, the CoPs-stimulated MC3T3-E1cells were transfected with si-TUG1 or co-transfected with si-TUG1 and si-BMP-7. The western blot result showed that the co-transfection with si-TUG1 and si-BMP-7 reduced the enhancement of si-TUG1 treatment alone (Figure 5A). The result of flow cytometry demonstrated that cell apoptosis was restored by the co-transfection with si-TUG1 and si-BMP-7 accompanied with the restore of caspase-3 protein level (Figure 5B). Additionally, knockdown of TUG1 and BMP-7 reversed the increase of cell viability raised by si-TUG1 (Figure 5C).

\section{Knockdown of TUG1 increased the BMD level of the osteolytic mouse model}

The osteolytic mouse model was established and was injected with lentivirus carrying si-TUG1 or si-control. After 14 days, the bone tissues were collected and the BMD level was detected. The results revealed that TUG1 knockdown significantly increased the BMD level compared with lv-si-control (Figure 6A). Then the real-time PCR and western blot confirmed the down-regulation of TUG1 and the up-regulation of BMP-7 (Figure $6 \mathrm{~B}$ and $6 \mathrm{C})$.

\section{DISCUSSION}

Recently, the mechanism of osteolysis has been reported by various studies. It has been reported that wear debris could induce aseptic loosing and osteolysis ${ }^{[13-}$ ${ }^{15]}$. Thus, in the present study, the clinical patients of hip arthroplasty with or without aseptic loosing were enrolled for studying the mechanism of osteolysis. Our findings identified that TUG1 was increased, while BMP-7 was decreased in periprosthetic tissues of hip arthroplasty in comparison with the normal tissues, in- dicating that TUG1 and BMP-7 might serve an important role in periprosthetic osteolysis.

It has been reported that many lncRNAs have been reported to be involved in osteolysis. For example, lncRNA KCNQ10T1 was identified to promote osteogenic differentiation to relieve osteolysis via activating Wnt/ $\beta$-cateinin ${ }^{[16]}$. LncRNA TSIX promoted osteoblast apoptosis in particle-induced osteolysis ${ }^{[3]}$. In the present study, high level of TUG1 served as an important marker of periprosthetic osteolysis in hip arthroplasty.

LncRNA is reported to mediate physiologic processes via regulating proteins. For example, IncRNA DGCR5 targets PRDM5 to regulate neuronal apoptosis in acute spinal cord injury ${ }^{[17]}$. LncRNA THOR mediated the progression of retinoblastoma through regulating c-myc and IGF2BP1 ${ }^{[18]}$. In addition, TUG1 has been reported to target YAP to regulate cell proliferation and migration of renal cell carcinoma ${ }^{[19]}$. In the present study, our result revealed that TUG1 mediated periprosthetic osteolysis by directly targeting BMP-7.

It has been reported that the signaling protein BMP-7 showed a significant role in the development of mammalian organs, such as the kidney and the eye ${ }^{[20]}$. BMP7 could regulate receptor-regulated Smads (Smad1, Smad5, and Smad8) and inhibitory Smads (Smad6 and Smad7) by binding to the type I and II receptors in a complex wound-healing signaling network ${ }^{[21]}$. In addition, BMP-7 was identified to regulate cell apoptosis of keratocyte ${ }^{[21]}$, mouse metanephric mesenchymal cells (29247399), and nucleus pulposus cells ${ }^{[22]}$. In the present study, BMP-7 was identified to regulate cell apoptosis of particle-stimulated osteoblasts, with the potential mechanism of knockdown of TUG1 suppressing osteoblast apoptosis by up-regulating BMP-7.
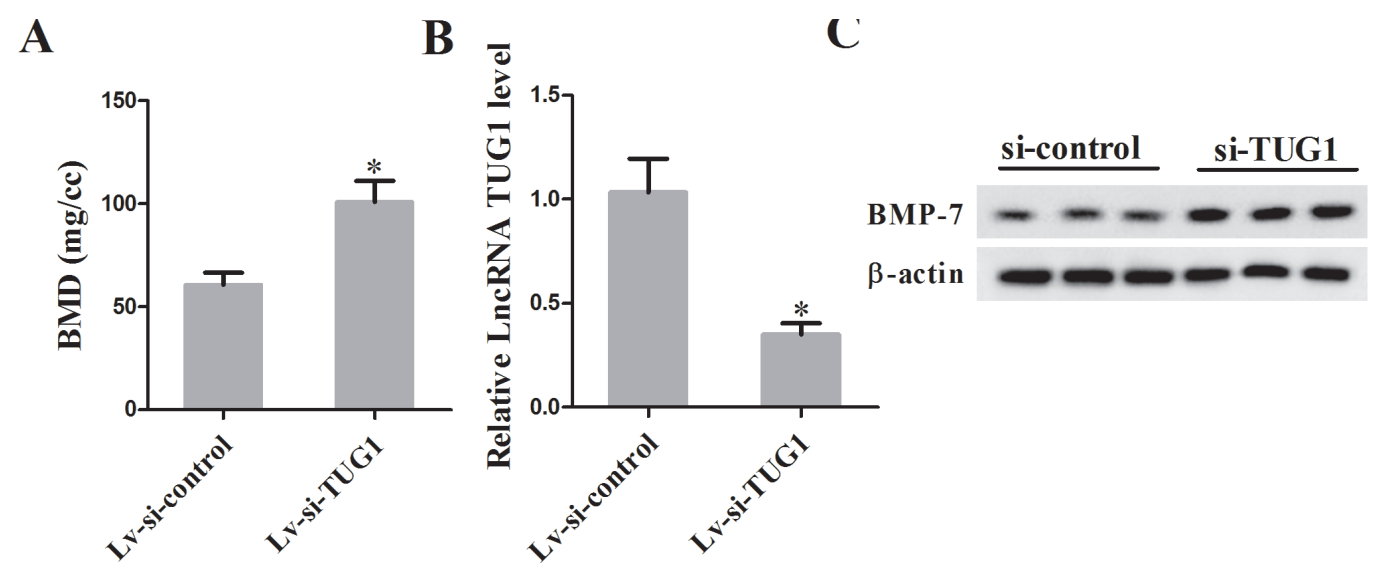

Figure 6. Knockdown of TUG1 increased the BMD level of the osteolytic mouse model. A total of 16 mice was divided into the lv-si-control and the lv-si-TUG1 group (n=8 in each group). (A) The BMD level of the two groups. (B) The expression of TUG1 was determined using real-time PCR. (C) The expression of BMP-7 was detected using western blot. *p<0.05 vs si-control. 
In conclusion, our findings demonstrated that increased TUG1 and decreased BMP-7 were detected in periprosthetic tissues of hip arthroplasty and particle-induced osteoblasts. The in vitro study identified that TUG1 mediated particle-induced osteoblast apoptosis by regulating the expression of BMP-7, which provided a new insight for the therapy of periprosthetic osteolysis in hip arthroplasty.

\section{REFERENCES}

1. Sukur, E., Akman, Y. E., Ozturkmen, Y., and Kucukdurmaz, F. (2016) Particle Disease: A Current Review of the Biological Mechanisms in Periprosthetic Osteolysis After Hip Arthroplasty. Open Orthop J 10, 241-251

2. Haynes, D. R., Crotti, T. N., Potter, A. E., Loric, M., Atkins, G. J., Howie, D. W., and Findlay, D. M. (2001) The osteoclastogenic molecules RANKL and RANK are associated with periprosthetic osteolysis. J Bone Joint Surg Br 83, 902911

3. Bu, Y., Zheng, D., Wang, L., and Liu, J. (2017) LncRNA TSIX promotes osteoblast apoptosis in particle-induced osteolysis by down-regulating miR-30a-5p. Connect Tissue Res, 1-8

4. Valladares, R. D., Nich, C., Zwingenberger, S., Li, C., Swank, K. R., Gibon, E., Rao, A. J., Yao, Z., and Goodman, S. B. (2014) Toll-like receptors-2 and 4 are overexpressed in an experimental model of particle-induced osteolysis. J Biomed Mater Res A 102, 3004-3011

5. Tang, Z., Gong, Z., and Sun, X. (2018) LncRNA DANCR involved osteolysis after total hip arthroplasty by regulating FOX01 expression to inhibit osteoblast differentiation. J Biomed Sci 25, 4

6. Gong, Z. M., Tang, Z. Y., and Sun, X. L. (2018) LncRNA PRNCR1 regulates CXCR4 expression to affect osteogenic differentiation and contribute to osteolysis after hip replacement. Gene 673, 251-261

7. Young, T. L., Matsuda, T., and Cepko, C. L. (2005) The noncoding RNA taurine upregulated gene 1 is required for differentiation of the murine retina. Curr Biol 15, 501512

8. Ma, B., Li, M., Zhang, L., Huang, M., Lei, J. B., Fu, G. H., Liu, C. X., Lai, Q. W., Chen, Q. Q., and Wang, Y. L. (2016) Upregulation of long non-coding RNA TUG1 correlates with poor prognosis and disease status in osteosarcoma. Tumour Biol 37, 4445-4455

9. Han, Y., Liu, Y., Gui, Y., and Cai, Z. (2013) Long intergenic non-coding RNA TUG1 is overexpressed in urothelial carcinoma of the bladder. J Surg Oncol 107, 555-559

10. Kim, Y., Kang, B. J., Kim, W. H., Yun, H. S., and Kweon, O. K. (2018) Evaluation of Mesenchymal Stem Cell Sheets Overexpressing BMP-7 in Canine Critical-Sized Bone Defects. Int J Mol Sci 19

11. ten Dijke, P., Korchynskyi, O., Valdimarsdottir, G., and Goumans, M. J. (2003) Controlling cell fate by bone mor- phogenetic protein receptors. Mol Cell Endocrinol 211, 105-113

12. Kureel, J., Dixit, M., Tyagi, A. M., Mansoori, M. N., Srivastava, K., Raghuvanshi, A., Maurya, R., Trivedi, R., Goel, A., and Singh, D. (2014) miR-542-3p suppresses osteoblast cell proliferation and differentiation, targets BMP-7 signaling and inhibits bone formation. Cell Death Dis 5, e1050

13. Tsertsvadze, A., Grove, A., Freeman, K., Court, R., Johnson, S., Connock, M., Clarke, A., and Sutcliffe, P. (2014) Total hip replacement for the treatment of end stage arthritis of the hip: a systematic review and meta-analysis. PLoS One 9, e99804

14. Epinette, J. A., and Manley, M. T. (2014) No differences found in bearing related hip survivorship at 10-12 years follow-up between patients with ceramic on highly cross-linked polyethylene bearings compared to patients with ceramic on ceramic bearings. J Arthroplasty 29, 1369-1372

15. Lopez-Lopez, J. A., Humphriss, R. L., Beswick, A. D., Thom, H. H. Z., Hunt, L. P., Burston, A., Fawsitt, C. G., Hollingworth, W., Higgins, J. P. T., Welton, N. J., Blom, A. W., and Marques, E. M. R. (2017) Choice of implant combinations in total hip replacement: systematic review and network meta-analysis. BMJ 359, j4651

16. Gao, X., Ge, J., Li, W., Zhou, W., and Xu, L. (2018) LncRNA KCNQ10T1 promotes osteogenic differentiation to relieve osteolysis via Wnt/beta-catenin activation. Cell Biosci 8, 19

17. Zhang, H., Wang, W., Li, N., Li, P., Liu, M., Pan, J., Wang, D., Li, J., Xiong, Y., and Xia, L. (2018) LncRNA DGCR5 suppresses neuronal apoptosis to improve acute spinal cord injury through targeting PRDM5. Cell Cycle 17, 19922000

18. Shang, Y. (2018) LncRNA THOR acts as a retinoblastoma promoter through enhancing the combination of c-myc mRNA and IGF2BP1 protein. Biomed Pharmacother 106, 1243-1249

19. Liu, S., Yang, Y., Wang, W., and Pan, X. (2018) Long noncoding RNA TUG1 promotes cell proliferation and migration of renal cell carcinoma via regulation of YAP. J Cell Biochem 119, 9694-9706

20. Dudley, A. T., Lyons, K. M., and Robertson, E. J. (1995) A requirement for bone morphogenetic protein-7 during development of the mammalian kidney and eye. Genes Dev 9, 2795-2807

21. Tandon, A., Sharma, A., Rodier, J. T., Klibanov, A. M., Rieger, F. G., and Mohan, R. R. (2013) BMP7 gene transfer via gold nanoparticles into stroma inhibits corneal fibrosis in vivo. PLoS One 8, e66434

22. Li, X., Cheng, S., Wu, Y., Ying, J., Wang, C., Wen, T., Bai, X., Ji, W., Wang, D., and Ruan, D. (2018) Functional self-assembled peptide scaffold inhibits tumor necrosis factor-alpha-induced inflammation and apoptosis in nucleus 
